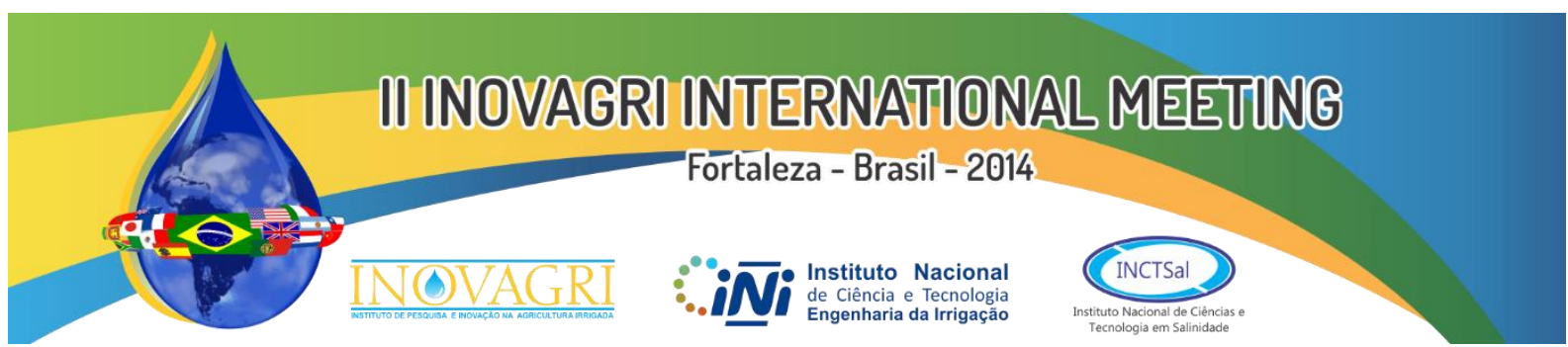

http://dx.doi.org/10.12702/ii.inovagri.2014-a567

\title{
CONCENTRAÇÃO DA SOLUÇÃO DE INJEÇÃO E FREQUÊNCIA DE FERTIRRIGAÇÃO EM LATOSSOLO AMARELO
}

\author{
J. de C. Teixeira ${ }^{1}$, E. F. Coelho ${ }^{2}$ D. L. Barros ${ }^{1}$
}

\begin{abstract}
RESUMO: A concentração da solução de injeção tem sido pouco considerada na fertirrigação. Entretanto ela pode influenciar o estado salino do solo, com risco de aumentos instantâneos da salinidade, que pode com o tempo afetar as características químicas do solo, além de induzir problemas de entupimentos nos dutos de condução de água para as plantas. O trabalho objetivou definir o binômio concentração da solução de injeção e frequência de fertirrigação, isto é, definir a concentração da solução de injeção mais adequada para duas frequências de fertirrigação para a bananeira Grand Naine. O experimento foi realizado em solo de textura argilosa de Tabuleiro Costeiro com um delineamento experimental em blocos casualizados, em esquema fatorial $3 \times 2$ sendo três concentrações da solução de injeção $(3,10$ e $15 \mathrm{~g} / \mathrm{L})$ x duas frequências de fertirrigação $(3$ e 7 dias). Foram avaliadas variáveis de crescimento e produção da bananeira e a condutividade elétrica do extrato de saturação (CEes) na zona radicular durante o ciclo da cultura. Os resultados mostraram que a faixa de concentração da solução injetora utilizada não acarretou elevação na CEes do solo suficiente para causar problemas de salinidade no mesmo ao longo do ciclo da bananeira. As diferentes concentrações e frequências de aplicação da solução injetora não tiveram efeito sobre a produtividade da cultivar estudada.
\end{abstract}

PALAVRAS CHAVE: bananeira, condutividade elétrica, salinidade

\section{CONCENTRATION OF INJECTION SOLUTION AND FERTIRRIGATION FREQUENCY IN YELLOW LATOSSOL}

ABSTRACT: The concentration of injection solution has been shortly considered in fertirrigation. However it might influence the saline status of the soil with risk for transient increase of soil salinity that may affect soil chemical characteristics along time. Moreover it may cause clogging problems inside pipes and hoses the conduct wter to plants. The work had as objective to define the binomial concentration of injection solution and fertirrigation frequency, i.e., to define the concentration of injection solution more adequate for two fertirrigation frequencies for Grand Naine banana. The experiment was carried in a clay soil of coastal tableland with an experimental design as randomized blocks in a $3 \times 2$ factorial scheme, with three concentrations of injection solution $(3,10$ e $15 \mathrm{~g} / \mathrm{L}) \mathrm{x}$ two fertirrigation frequencies ( 3 and 7 days). Banana growth and production variables were evaluated as well electrical conductivity of saturation extract (CEes) in the root zone during the crop cycle. Results showed that the range of injection solution concentration did not raise CEes enough to cause salinity problems in the soil alog the banana cycle. The different concentrations and frequencies of injection solution application did not have effect on yield of the studied cultivar.

KEY WORDS: banana, electrical conductivity, salinity

\section{INTRODUÇÃO}

A banana é uma das frutas mais consumidas no mundo. Seu cultivo necessita de manejo adequado. Nos últimos anos, a forma tradicional de adubação em cultivos irrigados vem sendo substituída pela aplicação de fertilizantes via água de irrigação (Sousa et al., 2002). A fertirrigação, entretanto não tem seguido critérios que favoreçam a sustentabilidade do ambiente solo, dada a deficiência de conhecimento técnico dos irrigantes, que desconhecem as bases da tecnologia. Concentrações elevadas

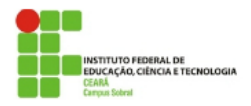


de sais na solução injetora aplicadas em alta frequência podem causar problemas de entupimento de emissores de irrigação e elevar a salinidade do solo. A utilização inadequada de fertilizantes gera perdas de nutrientes causadas pela erosão e/ou lixiviação. Tal fato tem grande importância para o produtor agrícola, pelo fato de significar baixas eficiências de utilização de nutrientes pelas culturas e, por consequência menores rendimentos (Konrad, 2002). O uso de fertilizante deve presidir o manejo racional dos nutrientes agregados, permitindo obter níveis ótimos de produtividade e ao mesmo tempo minimizar o impacto ambiental (Duggan, 2005). Uma das maiores preocupações na agricultura irrigada tem sido a aplicação indevida de fertilizantes com consequências negativas aos lençóis freáticos, principalmente pela lixiviação de nitratos. Os níveis de nitrato na água devem ser inferiores a $10 \mathrm{mgL}^{-1}$, enquanto no solo até $400 \mathrm{mgL}^{-1}$,(Dimenstain,1999), são mais adequados às culturas. O monitoramento constante de $\mathrm{NO}_{3}{ }^{-}$na solução do solo torna-se imprescindível para um manejo racional da fertirrigação. O conhecimento da condutividade elétrica do solo é importante para verificar a disponibilidade de nutrientes, ao longo do ciclo de uma cultura e os impactos de aplicação de fertilizantes nos atributos químicos do solo. Os trabalhos de avaliação de impactos de fontes nitrogenadas no $\mathrm{pH}$ do solo têm-se voltado para fontes amidicas e amoniacais como a uréia, o sulfato de amônio e fontes nítricas como o nitrato de cálcio (Alves et al., 2006), entretanto nesses trabalhos a concentração dessas fontes tem sido constante na água de irrigação. Estudos avaliando efeito da concentração de sais referentes a fontes nitrogenadas sobre o comportamento do $\mathrm{pH}$ e da condutividade elétrica no solo, ou no extrato de saturação do solo ainda não são conclusivos (Carvalho et al., 2009). Objetivou-se avaliar os efeitos da concentração da solução injetora aplicada em duas frequências de fertirrigação nas varáveis de crescimento, produção e na condutividade elétrica do extrato de saturação.

\section{MATERIAL E MÉTODOS}

O experimento foi conduzido na área experimental da Embrapa Mandioca e Fruticultura, em Cruz das Almas, Bahia. A cultura usada foi a bananeira cultivar Grande Naine plantada no espaçamento 2,0 $\mathrm{m} \times 2,5 \mathrm{~m}$. O delineamento experimental foi em blocos casualizados com seis tratamentos e quatro repetições, com dez plantas por parcela sendo quatro utilizadas como úteis. $\mathrm{O}$ experimento seguiu um esquema fatorial $3 \times 2$ sendo três concentrações da solução de injeção x duas frequências de fertirrigação com quatro repetições. As concentrações da solução de injeção foram de 3, 10 e $15 \mathrm{~g} / \mathrm{L}$, valores esses, definidos a partir de resultados de trabalhos executados em solo franco argiloso alico de Tabuleiros costeiros (Carvalho et. al., 2009; Coelho et. al., 2010). As frequências de fertirrigação foram de 3 e 7 dias no primeiro ano e 7 e 15 dias no segundo ano que são frequências recomendadas para fertirrigação da bananeira (Borges et. al., 2009; Coelho et. al., 2009). Ou seja, T1 = concentração de $3 \mathrm{~g} / \mathrm{l}$ e frequência de 7 dias; T2 = concentração de $10 \mathrm{~g} / \mathrm{l}$ e frequência de 7 dias; T3 = concentração de $15 \mathrm{~g} / \mathrm{l}$ e frequência de 7 dias; T4 = concentração de $3 \mathrm{~g} / \mathrm{l}$ e frequência de 3 dias; T5 = concentração de $10 \mathrm{~g} / \mathrm{l}$ e frequência de 3 dias; $\mathrm{T} 6=$ concentração de $15 \mathrm{~g} / \mathrm{l}$ e frequência de 3 dias. O nitrogênio $(\mathrm{N})$ foi aplicado de acordo com recomendação de Borges \& Costa (2002), com base no valor de 270 Kg.ha- ${ }^{1}$. ano- ${ }^{-1}$ em dois ciclos da cultura. Além do nitrogênio, foi aplicado potássio $\left(\mathrm{K}_{2} \mathrm{O}\right)$ via água de irrigação seguindo recomendação de Borges \& Costa (2002). Ambos os nutrientes foram aplicados na forma de nitrato de potássio. Foi utilizada irrigação por microaspersão com um emissor de $60 \mathrm{~L} / \mathrm{h}$ para quatro plantas. As irrigações foram realizadas com base no balanço de água do solo simplificado (Coelho et. al., 2003), tendo leituras de TDR como indicadores do momento da irrigação. Foram avaliados em uma planta de cada parcela experimental as variaveis biométricas: altura e diâmetro do pseudocaule (com auxilio de uma régua graduada e uma fita métrica respectivamente), número de dias para colheita e número de folhas vivas numa frequência de 30 dias. As variáveis de produção foram: peso de cacho, peso de pencas, número de frutos/cacho, peso médio dos frutos, número de pencas e número de folhas vivas na colheita. Mensalmente, durante o ciclo da cultura foram retiradas, a 0,30 e $0,70 \mathrm{~m}$ de profundidade, próximo ao ponto de emissão do gotejador a $0,30 \mathrm{~m}$ do pseudocaule, amostras de solo para avaliação do extrato de saturação para a determinação da condutividade elétrica do extrato de saturação e da concentração de $\mathrm{NO}_{3}$ (nitrato). A condutividade elétrica do extrato de saturação (CES) foi determinada usando um condutivímetro de mesa e a concentração de nitrato foi determinada com uso de kits rápido Card Horiba. Os dados biométricos e de produção foram 
submetidos a analise de variância. Os dados da condutividade elétrica do extrato de saturação foram avaliados para o período de um ciclo da cultura da bananeira.

\section{RESULTADOS E DISCUSSÃO}

A análise de variância mostrou que os tratamentos não foram significativos para as variáveis em estudo. No entanto, o tratamento 6 apresentou maior número de folhas (15), altura de plantas $(2,41 \mathrm{~m})$ (Tabela 1). Apresentou, ainda, maior número de frutos (181), comprimento do fruto central da segunda penca $(24,65 \mathrm{~cm})$, produtividade de penca $\left(70,62 \mathrm{t} \mathrm{ha}^{-1}\right)$ e produtividade cacho $\left(75,94 \mathrm{t} \mathrm{ha}^{-1}\right)$ ( Tabela 2). O tratamento 5 juntamente com o 4 apresentaram maior diâmetro de pseudocaule com $23,75 \mathrm{~cm}$ (Tabela 1). Este último apresentou também, maior número de folhas na colheita e maior número de pencas por cacho ( Tabela 2).

Tabela1. Médias do número de folhas, da altura da planta e do diâmetro do pseudocaule, no momento da emissão da inflorescência da bananeira cv. Grand Naine.

\begin{tabular}{cccc}
\hline & NÚMERO & \multicolumn{2}{c}{ PSEUDOCAULE } \\
TRATAMENTO & DE FOLHA & ALTURA(m) & $\begin{array}{l}\text { DIÂMETRO } \\
(\mathrm{cm})\end{array}$ \\
\hline 1 & 14 & 2,33 & 23,00 \\
2 & 15 & 2,43 & 22,25 \\
3 & 14 & 2,33 & 22,25 \\
4 & 14 & 2,36 & 23,75 \\
5 & 14 & 2,38 & 23,75 \\
6 & 15 & 2,41 & 22,75 \\
\hline $\mathrm{CV}(\%)$ & 3,06 & 6,17 & 6,39 \\
\hline
\end{tabular}

Tabela 2. Médias das variáveis de produção da bananeira cv. Grand Naine, sob diferentes concentrações da solução de injeção e frequência de fertirrigação.

\begin{tabular}{cccccccc}
\hline $\begin{array}{c}\text { TRATA } \\
\text { MENTO }\end{array}$ & NFOLHAS & $\begin{array}{c}\text { NFRUTO } \\
\text { S }\end{array}$ & NPENCAS & $\begin{array}{c}\text { COMP } \\
(\mathrm{cm})\end{array}$ & $\begin{array}{c}\text { DIAM } \\
(\mathrm{mm})\end{array}$ & $\begin{array}{c}\text { PPENCA } \\
\left(\text { tha }^{-1}\right)\end{array}$ & $\begin{array}{c}\text { PCACHO } \\
\left(\mathrm{tha}^{-1}\right)\end{array}$ \\
\hline 1 & 9,87 & 165,44 & 9,06 & 23,50 & 38,50 & 64,33 & 69,07 \\
2 & 9,81 & 159,68 & 9,12 & 23,75 & 38,50 & 66,59 & 71,50 \\
3 & 10,25 & 169,31 & 9,12 & 23,62 & 38,44 & 63,19 & 68,52 \\
4 & 11,12 & 179,81 & 9,62 & 23,00 & 37,50 & 67,74 & 72,75 \\
5 & 9,56 & 169,25 & 9,50 & 21,75 & 36,56 & 63,50 & 68,39 \\
6 & 10,12 & 181,81 & 9,56 & 24,65 & 38,44 & 70,62 & 75,94 \\
\hline CV $(\%)$ & 9,88 & 11,44 & 7,06 & 6,71 & 4,13 & 16,17 & 15,96 \\
\hline
\end{tabular}

NFOLHAS $=$ número de folhas; NFRUTOS $=$ número de frutos/cacho; NPENCAS $=$ número de pencas/cacho; $\mathrm{COMP}=$ comprimento do fruto central da segunda penca; DIAM= diâmetro do fruto central da segunda penca; $\mathrm{PPENCA}=$ produtividade de pencas; $\mathrm{PCACHO}=$ produtividade de cachos.

Esses valores foram superiores aos encontrados por Alves et al. (2010) o qual estudando diferentes combinações e duas fontes nitrogenadas, uréia e nitrato de cálcio, aplicadas por fertirrigação sobre o crescimento e a produtividade da bananeira Grande registraram variação de 19,17 a 21,1 t ha ${ }^{-1}$ para produtividade de pencas. Sendo o maior valor apresentado por tratamento utilizando apenas nitrato de cálcio. Esse tratamento demonstrou, ainda, maior comprimento $(29,8 \mathrm{~cm})$ e diâmetro de fruto (37,4 mm) par o primeiro ciclo de produção. Barros et al. (2011) encontrou altura 2,63 m e 24,3 $\mathrm{cm}$ de diâmetro do pseudocaule para tratamento que usava nitrato de cálcio durante todo o ciclo. Além disso, também se destacou o comprimento do fruto central da segunda penca $(21,5 \mathrm{~cm})$ quando usado esse tratamento.

A CEes (condutividade elétrica do extrato de saturação) média ao longo do ciclo variou de 0,06 a $5,97 \mathrm{dS} \mathrm{m}^{-1}$ (Figura 1) considerando todos os tratamentos para a profundidade de $0,30 \mathrm{~m}$. A maioria dos valores ficou abaixo de $3,5 \mathrm{dS} \mathrm{m}^{-1}$ considerando todo o período observado, sendo que entre junho e dezembro a CEes ficou abaixo de $2,1 \mathrm{dS} \mathrm{m}^{-1}$. 
Para a profundidade $0,70 \mathrm{~m}$, no período de dezembro a junho, os tratamentos de maior concentração da solução de injeção ou da água durante a fertirrigação resultaram em maiores CEes seguido pelos tratamentos de menor concentração da água na fertirrigação, com valores abaixo de 3,7 $\mathrm{dSm}^{-1}$. Esses resultados evidenciam coerência entre as concentrações da água de irrigação e as CEes do solo. No período de junho a dezembro a CEes ficou abaixo de $1,1 \mathrm{dSm}^{-1}$ para todos os tratamentos. Esses resultados mostram que a concentração da solução de injeção e consequentemente da água de irrigação tem efeito na salinidade do solo, mesmo que temporariamente. Os períodos de elevação da CEes, apesar de corresponder próximo de seis meses não foram suficientes para influenciar o crescimento ou a produção da cultura, o que se deve a variação da CEes nesse período com valores por exemplo de 3,5 $\mathrm{dSm}^{-1}$ num dia e de $1,5 \mathrm{dSm}^{-1}$ noutro dia (Figuras 1 e 2). Dessa forma na maior parte do período os valores ficaram próximos ou abaixo da CEes critica de 1,1 dS m-1 (OLIVEIRA et al., 1999). Esses resultados são concordantes com os obtidos por COSTA et al. (2009) que não verificaram variações relevantes na CEes do solo para concentrações de uréia e nitrato de potássio na água de irrigação até $7,2 \mathrm{mgL}^{-1}$
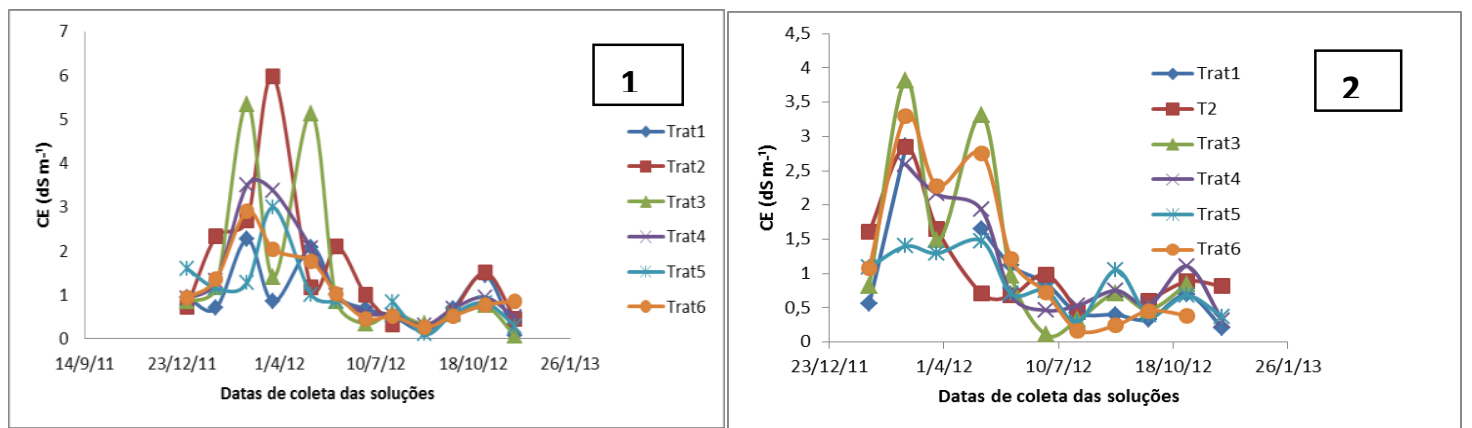

Figura 1. Condutividade elétrica no extrato de saturação na profundidade de $30 \mathrm{~cm}$ em fertirrigação com diferentes concentrações da solução injetora e frequência de aplicação.

Figura 2. Condutividade elétrica no extrato de saturação na profundidade de $0,70 \mathrm{~m} \mathrm{em}$ fertirrigação com diferentes concentrações da solução injetora e frequência de aplicação.

As médias de CEs apresentaram variações superiores para as profundidades de $0,30 \mathrm{~m}$ quando comparadas a de $0,70 \mathrm{~m}$. Esse resultado concorda com os encontrados por Peixoto et al., (2006), que observaram aumento na CEes da camada mais superficial, e a literatura tem mostrado valores absolutos superiores para a camada 0,30 m onde ocorre constante presença dos íons resultantes das fontes de $\mathrm{N}$ e onde ocorre mais acentuada dinâmica desses íons (Rolston et al, 1986) comparada a profundidade de $0,70 \mathrm{~m}$. Na maioria do ciclo da bananeira, os valores de CEes ficaram abaixo dos limites dos solos considerados salinos estabelecido tanto pela classificação da U. S. Salinity Laboratory $\left(4,0 \mathrm{dS} . \mathrm{m}^{-1}\right)$, em como próximo ou abaixo do limite estabelecido pelo Comitê de Terminologia da Sociedade Americana de Ciência do Solo (2,0 dS.m-1).

As concentrações de nitrato $\left(\mathrm{NO}_{3}^{-}\right)$ao longo do tempo no extrato de saturação foram iguais ou inferiores a $600 \mathrm{mg} . \mathrm{L}^{-1}$ para todos os tratamentos, exceto para o tratamento 3 a $0,30 \mathrm{~m}$ da superfície do solo (Figura 3). De modo geral, os tratamentos 4 e 5 foram os de menor variação de $\mathrm{NO}_{3}{ }^{-}$ao longo do ciclo (Figura 3). Os valores da concentração de $\mathrm{NO}_{3}{ }^{-}$na maioria do ciclo da cultura ficaram abaixo de $600 \mathrm{mg}$. L-1 $^{1}$ para as duas profundidades (Figuras 3 e 4), sendo que no período de junho a dezembro ficaram abaixo de $200 \mathrm{mg}$.L-1 ${ }^{-1}(0,30 \mathrm{~m})$ e de $120 \mathrm{mg}$.L-1 $(0,70 \mathrm{~m})$. 

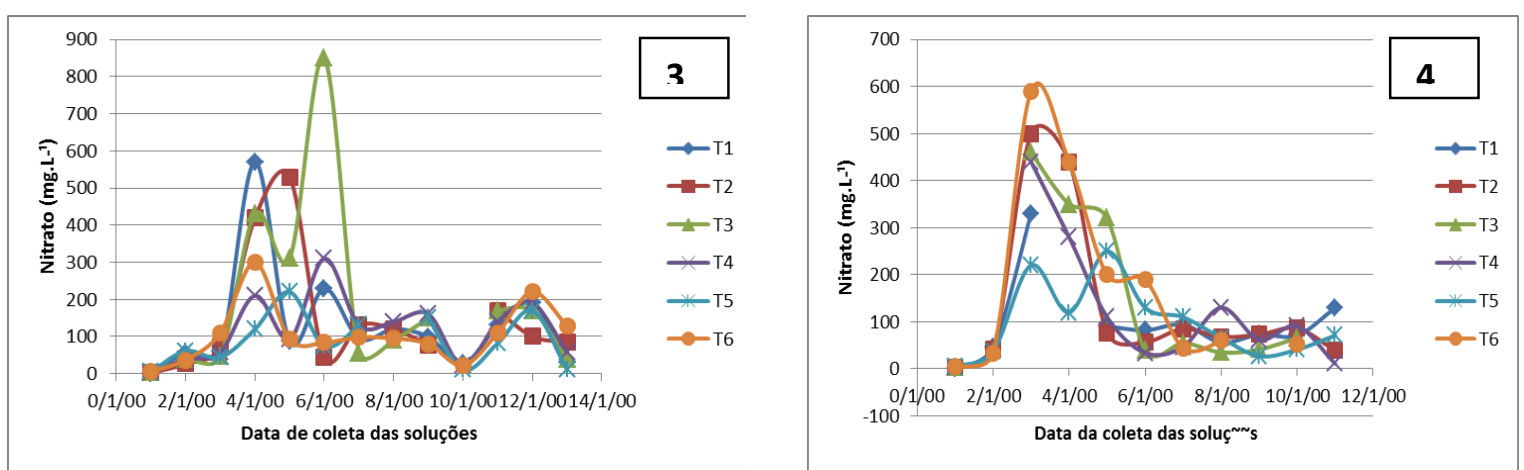

Figura 3. Teores de $\mathrm{NO}_{3}{ }^{-}$no extrato de saturação na profundidade de $0,30 \mathrm{~m}$ em fertirrigação com diferentes concentrações da solução injetora e frequência de aplicação.

Figura 4. Teores de NO3 no extrato de saturação na profundidade de $70 \mathrm{~cm}$ em fertirrigação com diferentes concentrações da solução injetora e frequência de aplicação.

\section{CONCLUSÕES}

Não houve estatisticamente nenhum efeito das diferentes concentrações e frequências de aplicação da solução injetora sobre o crescimento e produtividade da cultivar estudada.

As concentrações de nitrato no extrato de saturação durante o ciclo da bananeira estiveram dentro dos limites aceitáveis (abaixo de $400 \mathrm{mgL}^{-1}$ ).

\section{REFERÊNCIAS}

ALVES, M.S. aplicação de diferentes combinações de fontes nítrica e amídica via fertirrigação na bananeira "grand naine" e seus efeitos no solo e na cultura 2006. 52f. Dissertação (Mestrado em Ciências Agrárias). Universidade Federal da Bahia, Bahia.

ALVES, M. da S.; coelho, E. F.; PAZ, V. P. da S.; NETO, T. M. de A. Crescimento e produtividade da bananeira cv. Grande Naine sob diferentes combinações de nitrato de cálcio e ureia. Rev. Ceres, Viçosa, v. 57, n.1, p. 125-131, jan/fev, 2010.

ANDRADE NETO, T. M.; Coelho, E. F; Santana, J. A do. ; SANTANA JUNIOR, E. B; Alves, $\mathrm{M}$ da S. Efeito da concentração de nitrato de cálcio e uréia na água de irrigação via gotejamento na salinidade do solo. In: Anais... II Workshop Internacional de Inovações Tecnologicas na Irrigação \& I Simposio Brasileiro sobre uso Multiplo da Água., 2008, Fortaleza.

BARROS, D. L.; COELHO, E. F.; AZEVEDO, N. F. de.; PAMPONET, A. J. M. Produtividade da bananeira cv. grand naine fertirrigada por diferentes combinações de nitrato de cálcio e uréia. In: XXI Congresso nacional de irrigação, Petrolina - PE, 2011. Congresso nacional de irrigação - as oportunidades de empreendedorismo na agricultura irrigada, 2011.

BORGES, A.L.; SILVA, J.T.A. da; OLIVEIRA, S.L. de. Adubação nitrogenada e potássica para bananeira cv. Prata-Anã irrigada: produção e qualidade dos frutos no primeiro ciclo. Revista Brasileira de Fruticultura, Jaboticabal-SP, v.19, n.2,p.179-84. 1997.

BORGES, A.L.; SILVA, D.J. Fertilizantes para fertirrigação. In: BORGES, A.L.; COELHO, E.F.; TRINDADE, A.V. (Org.). Fertirrigação em fruteiras tropicais. Cruz das Almas: Embrapa Mandioca e Fruticultura, 2002. p.15-27.

BURGUEÑO, H. La fertirrigacion en cultivos hortícolas com acolchado plástico. Culiacan, 1996. v.1, 45p.

CERETTA, C.A. Manejo da adubação nitrogenada na sucessão aveia/milho, no sistema plantio direto. In: CURSO DE ATUALIZAÇÃO EM RECOMENDAÇÕES DE ADUBAÇÃO E CALAGEM, ÊNFASE EM PLANTIO DIRETO, 3., Santa Maria, 1997. Anais. Santa Maria, p.112-124.

CARVALHO, G. C. ; COELHO, E. F. ; COSTA, F. S. ; BARBOSA, R. P. ; NASCIMENTO JUNIOR, A. L. ; AZEVEDO, N. F. . pH do solo no primeiro ciclo da bananeira da terra 
fertirrigada com diferentes concentrações de uréia e nitrato de potássio na água de irrigação. In: XXXII Congresso Brasileiro de Ciência do Solo, 2009, Fortaleza - CE. XXXII Congresso Brasileiro de Ciência do Solo - O solo e a produção de bioenergia: perspectivas e desafios, 2009.

DIMENSTEIN, L. fertirrigação. Fortaleza: Instituto Frutal, 1999. 31 p. Curso Técnico.

DUGGAN, M. T. Fertilizar eficientemente para reducir el riesgo ambiental: nitrógeno. Disponível em: http://fertitec.com/informaciones /fer_princ_fac_apl.htm/. Acesso em 30 agosto de 2007.

GOMES, J. A. Absorção de nutrientes pela bananeira cv. Prata (Musa AAB, subgrupo prata) em diferentes estádios de desenvolvimento, 1988. 98 p. Dissertação (Mestrado em Fruticultura), Escola Superior de Agricultura Luis de Queiroz, Universidade de São Paulo, Piracicaba.

KONRAD, M. Efeito de sistemas de irrigação localizada sobre a produção e qualidade da acerola (Malpighia spp) na região da Nova Alta Paulista. Ilha Solteira, Faculdade de Engenharia, 2002. 119p. (Mestrado em Sistema de Produção - Faculdade de Engenharia de Ilha Solteira - UNESP).

LAHAV, E.; TURNER, D. Banana Nutrition. Bern, Switzerland: International Potash Institute, 1983. 62p. (IPI-Bulletin 7).

OLIVEIRA, S.L. Irrigação. In: Alves, J. (Org.) A cultura da banana: aspectos técnicos socioeconômicos e agroindustriais. Brasília: Embrapa Produção e Informação, 1997. p.317334.

OLIVEIRA, S. O. de; ALVES, E. J.; SHEPHERD, K.; DANTAS, J. L. L. Cultivares. In: ALVES, E. J. (Org.) A cultura da banana: aspectos técnicos, socioeconômicos e agroindustriais. 2.ed., Brasília: Embrapa-SPI / Cruz das Almas: Embrapa-CNPMF, 1999, p.85-105.

PEIXOTO, J. F. S.; GUERRA, H. O. C.; CHAVES, L. H. G. Alterações de atributos químicos do solo pela fertirrigação com nitrogênio e potássio. Rev... Agropecuária Técnica, v.27, n.2, p.69-76, 2006.

REICHARDT, K. Irrigação. In: A água em sistemas agrícolas. São Paulo: Manole, 1990. p.13955.

ROLSTON, D.E.; MILLER, R.J.; SCHULBACH, H. Management principles ln: NAKAYAMA, F.S.; BUCKS, D.A. Trickle irrigation for crop prodution. Amsterdam: Elsevier, 1986.p.317-345. http://dx.doi.org/10.1016/B978-0-444-42615-4.50017-5

SILVA, J. T. A. Adubação e nutrição da bananeira para o Norte de Minas. Belo Horizonte-MG: EPAMIG, 1994,.24p. ( Boletim Técnico, 46).

SOUZA, V. F.; COELHO, E. F. Manejo de fertirrigação em fruteiras. In: FOLEGATTI, M. V.; CASARINI, E.; BLANCO, F. F.; BRASIL, R. P. C.; RESENDE, R. S. (Coord.) Fertirrigação: citrus, flores, hortaliças. Guaíba:Agropecuária, 2001, p.289-317.

SOUSA, V.F. de; FOLEGATTI, M.V.; COELHO FILHO, M.A. \& FRIZZONE, J.A. Distribuição radicular do maracujazeiro sob diferentes doses de potássio aplicadas por fertirrigação. Revista Brasileira de Engenharia Agrícola e Ambiental, v.6, p.51-56, 2002. http://dx.doi.org/10.1590/S1415-43662002000100010

VANOTTI, M.B.; BUNDY, L.G. An alternative rationale for corn nitrogen fertilizer recommendations. Journal of Production Agriculture, Madison, v.7, n.2, p.243-249, 1994. http://dx.doi.org/10.2134/jpa1994.0243 